Canadian Journal of Physics

Revue canadienne de physique

\title{
Partial wave off-shell Coulomb amplitudes at excited-state energy
}

\begin{tabular}{|r|l|}
\hline Journal: & Canadian Journal of Physics \\
\hline Manuscript ID & cjp-2017-0677.R2 \\
\hline Manuscript Type: & Article \\
\hline Date Submitted by the Author: & 25 -Nov-2017 \\
\hline Complete List of Authors: & $\begin{array}{l}\text { Kharchenko, Vladyslav; Bogolyubov Institute for Theoretical Physics, } \\
\text { National Academy of Sciences of Ukraine, Department of nuclear and } \\
\text { quantum field theory }\end{array}$ \\
\hline $\begin{array}{r}\text { Is the invited manuscript for } \\
\text { consideration in a Special } \\
\text { Issue? : }\end{array}$ & $\begin{array}{l}\text { Coulomb transition matrix,, partial wave expansion,, Lippmann-Schwinger } \\
\text { equation,, Fock method,, analytical solution }\end{array}$ \\
\hline
\end{tabular}




\title{
Partial wave off-shell Coulomb amplitudes at excited-state energy
}

\author{
Vladyslav F. Kharchenko \\ Bogolyubov Institute for Theoretical Physics, National Academy of Sciences \\ of Ukraine, UA - 03143, Kyiv, Ukraine \\ E-mail: vkharchenko@bitp.kiev.ua
}

\begin{abstract}
Based on the integral representation of the three-dimensional Coulomb transition matrix elaborated by us formerly with the use of specific symmetry of the interaction in the fourdimensional Euclidean space introduced by Fock, the possibility of the analytical solving of the integral equation for the partial wave transition matrices at the excited bound state energy has been studied. New analytical expressions for the partial $s$-, $p$ - and $d$-wave Coulomb $t$-matrices for like-charged particles and the expression for the partial $d$-wave $t$-matrix for unlike-charged particles at the energy of the first excited bound state have been derived.
\end{abstract}

Keywords: Coulomb transition matrix, partial wave expansion, Lippmann-Schwinger equation, Fock method, analytical solution

PACS numbers: 03.65.-w, 03.65.Nk

\section{Introduction}

The Coulomb transition matrix, which in the momentum space is a function of the initial and final relative momenta and the energy, provides all information about the system of two charged particles with the Coulomb interaction [1]. The knowledge of the Coulomb transition matrix with the momenta off the energy shell is necessary when studing properties of the few-body atomic and nuclear systems containing charged particles by using the Faddeev [2,3] and Faddeev-Yakuboskii [4] integral equations. The separation of the main Coulomb singularity and the regularization of the integral equations for the three-body system, which contains charged particles, has been performed by Vesselova $[5,6]$ using the known Gorshkov's receipe for the system of two charged particles [7].

Presently, there are known several representations of the two-body Coulomb transition matrix [8-18]. The one-parameter integral representations for the Coulomb Green's function which take imediately into account the existing symmetry of the Coulomb system in Fock's four-dimensional Euclidean space [19] have been firstly obtained in the papers by Bratsev and Trifonov [10] and Schwinger [13]. The expressions for three-dimensional Coulomb transition matrix with the explicit removal of the singularities in the transfermomentum variable and the energy have been derived in the papers [15] (for the negative values of the energy $E<0$ ) and [16] (for zeroth and positive energies $E \geq 0$ ). The possibility of obtaining the expressions for partial wave two-body Coulomb transition matrix at the energy of the ground bound state in the analytical form was investigated in previous papers [20] ( in the case of the attractive interaction) and [21] (in the case of the repulsive interaction ).

This paper is devoted to the derivation of the analytical expressions for the partial wave Coulomb transition matrices at the energy of the first excited state of the two-body system. In Section 2 we begin the derivation leaning upon the integral representation of the three-dimensional Coulomb $t$-matrix obtained in [15]. On this basis in Section 3 we 
deduce the general formula for the partial wave Coulomb matrix $t_{l}\left(k, k^{\prime} ; E\right)$ at the energy $E<0$. In Section 4 we study the simplification of the expression for the partial wave Coulomb $t$-matrix at the energy of the first excited state of the two-body bound system. In Section 5, the analytical expressions for the partial $s$-, $p$ - and $d$-wave Coulomb transition matrices in the case of the repulsive interaction are displayed. Section 6 is devoted to the presentation of the analytical expressions for the partial $d$-wave transition matrix in the case of the attractive Coulomb interaction. The discussion of the obtained results, concluding remarks on the performed work and the plans of the future studing are given in Section 7.

\section{Three-dimensional Coulomb transition matrix at negative energy}

In the momentum space the three-dimensional Coulomb transition matrix $<\mathbf{k}|t(E)| \mathbf{k}^{\prime}>$, which describes the system of two charged particles, is given by the Lippmann-Schwinger equation

$$
\left.\left.<\mathbf{k}|t(E)| \mathbf{k}^{\prime}\right\rangle=\left\langle\mathbf{k}|v| \mathbf{k}^{\prime}\right\rangle+\int \frac{d \mathbf{k}^{\prime \prime}}{(2 \pi)^{3}}\left\langle\mathbf{k}|v| \mathbf{k}^{\prime \prime}\right\rangle \frac{1}{E-\frac{k^{\prime \prime 2}}{2 \mu}}<\mathbf{k}^{\prime \prime}|t(E)| \mathbf{k}^{\prime}\right\rangle,
$$

with the free term in the form of the matrix of the Coulomb interaction between the particles 1 and 2

$$
\left\langle\mathbf{k}|v| \mathbf{k}^{\prime}\right\rangle=\frac{4 \pi q_{1} q_{2}}{\left|\mathbf{k}-\mathbf{k}^{\prime}\right|^{2}} .
$$

and the kernel, that is defined by the potential of interaction and the free propagator. Here $q_{1}$ and $q_{2}$ are the charges of the particles that interact between themselves, $E$ is the energy of the relative motion of the particles, $\mu$ is the reduced mass of the particles, $\mathbf{k}$ and $\mathbf{k}^{\prime}$ are the variable relative momenta in the final and the initial states, $\hbar$ is reduced Planck's constant.

In the case of the relative energy $E=-\hbar^{2} \kappa^{2} / 2 \mu$ the three-dimensional Coulomb transition matrix has the form [15]

$$
\begin{gathered}
<\mathbf{k}|t(E)| \mathbf{k}^{\prime}>=\frac{8 \pi q_{1} q_{2} \kappa^{2}}{\left(k^{2}+\kappa^{2}\right)\left(k^{\prime 2}+\kappa^{2}\right) \sin \omega}\left\{\cot \frac{\omega}{2}-\pi \gamma \cos \gamma \omega-\gamma \sin 2 \gamma \omega \ln \left(\sin \frac{\omega}{2}\right)\right. \\
\left.+2 \pi \gamma c(\gamma) \cot \gamma \pi \sin \gamma \omega+\gamma \cos \gamma \omega x_{\gamma}(\omega)+2 \gamma^{2} \sin \gamma \omega y_{\gamma}(\omega)\right\},
\end{gathered}
$$

where

$$
\gamma=\frac{\mu q_{1} q_{2}}{\hbar^{2} \kappa}
$$

is the known dimensionless Coulomb parameter (the Sommerfeld parameter). The variable quantity $\omega$ in Eq.(3) denotes the angle between two 4-dimensional vectors in the fourdimensional Euclidean space introduced by Fock [19], the three-dimensional vectors $\mathbf{k}$ and $\mathbf{k}^{\prime}$ lie in the hyperplane, which is the stereographic projection of the unit sphere. The variable $\omega$ is defined by the following expression

$$
\sin ^{2} \frac{\omega}{2}=\frac{\kappa^{2}\left|\mathbf{k}-\mathbf{k}^{\prime}\right|^{2}}{\left(k^{2}+\kappa^{2}\right)\left(k^{\prime 2}+\kappa^{2}\right)} \quad, \quad 0 \leq \omega \leq \pi
$$

The functions $x_{\gamma}(\omega), y_{\gamma}(\omega)$ and $c(\gamma)$ in (3) are given by

$$
x_{\gamma}(\omega)=\int_{0}^{\omega} d \varphi \sin \gamma \varphi \cot \frac{\varphi}{2}, y_{\gamma}(\omega)=\int_{\omega}^{\pi} d \varphi \sin \gamma \varphi \ln \left(\sin \frac{\varphi}{2}\right),
$$




$$
c(\gamma)=\frac{1}{2}\left[1-\frac{1}{\pi} x_{\gamma}(\pi)\right] .
$$

The singularities in the energy are contained in the fourth term of the expression in the braces (3). They arise only in the case of the attractive Coulomb interaction potential (for oppositely charged particles with $q_{1} q_{2}<0$ ), when the Coulomb parameter $\gamma$ takes on the negative integer values $(\gamma=-n, n=1,2,3, \ldots)$ corresponding to the spectrum of the bound states of the two-particle system with the energies

$$
E_{n}=-\frac{\mu\left(q_{1} q_{2}\right)^{2}}{2 \hbar^{2} n^{2}}, \quad n=1,2, \cdots .
$$

The values $\kappa$ and the Coulomb parameter $\gamma$ which correspond to the energy (7) are equal to

$$
\kappa_{n}=\frac{\sqrt{-2 \mu E_{n}}}{\hbar}=\frac{\mu\left|q_{1} q_{2}\right|}{\hbar^{2} n}, \quad \gamma_{n}=\frac{\mu q_{1} q_{2}}{\hbar^{2} \kappa_{n}}=\frac{q_{1} q_{2}}{\left|q_{1} q_{2}\right|} n .
$$

For the energies of the bound states $E=E_{n}$ the Coulomb parameter $\gamma$ takes the integer values - positive for the repulsive Coulomb interaction $\left(q_{1} q_{2}>0, \gamma_{n}=n\right)$ and negative for the attractive interaction $\left(q_{1} q_{2}<0, \gamma_{n}=-n\right)$. Thus, taking into account that

$$
x_{n}(\pi)=\pi, \quad x_{-n}(\pi)=-\pi,
$$

we find from the expression for $c(\gamma)(6)$

$$
c(n)=0, \quad c(-n)=1 .
$$

In the case of the repulsive interaction $(\gamma>0)$ the expression for the three-dimensional Coulomb transition matrix (3) has no singularities at the energies of the bound states $E=E_{n}$. The indeterminateness in the fourth term in the braces of the type $\frac{0}{0}$ at $\gamma=n$ we evaluate according to l'Hospital rule

$$
\left[\frac{2 \pi \gamma c(\gamma)}{\tan \gamma \pi}\right]_{\gamma \rightarrow n}=2 n c^{\prime}(n) \equiv \rho_{n}
$$

where

$$
\rho_{n}=(-1)^{n}-2 n \ln 2-2 n \sum_{m=1}^{n} \frac{(-1)^{m}}{m} .
$$

\section{Partial wave transition matrices at the negative energy}

Developing the matrix elements of the Coulomb potential and the corresponding threedimensional transition matrix in Legendre polinomials $P_{l}(x)$

$$
\begin{gathered}
\left\langle\mathbf{k}|v| \mathbf{k}^{\prime}\right\rangle=\sum_{l=0}^{\infty}(2 l+1) v_{l}\left(k, k^{\prime}\right) P_{l}\left(\hat{\mathbf{k}} \cdot \hat{\mathbf{k}}^{\prime}\right), \\
\left\langle\mathbf{k}|t(E)| \mathbf{k}^{\prime}\right\rangle=\sum_{l=0}^{\infty}(2 l+1) t_{l}\left(k, k^{\prime} ; E\right) P_{l}\left(\hat{\mathbf{k}} \cdot \hat{\mathbf{k}}^{\prime}\right),
\end{gathered}
$$

where $\hat{\mathbf{k}}$ is the unit vector along the vector $\mathbf{k}$ and $\hat{\mathbf{k}} \cdot \hat{\mathbf{k}}^{\prime}=\cos \theta$, we write the one-dimensional integral equation for the partial wave transition matrix in the form

$$
t_{l}\left(k, k^{\prime} ; E\right)=v_{l}\left(k, k^{\prime}\right)+\int_{0}^{\infty} \frac{d k^{\prime \prime} k^{\prime 2}}{2 \pi^{2}} v_{l}\left(k, k^{\prime \prime}\right) \frac{1}{E-\frac{k^{\prime \prime 2}}{2 \mu}} t_{l}\left(k^{\prime \prime}, k^{\prime} ; E\right)
$$


The inhomogeneous term and the kernel of this equation are expressed the partial wave component of the Coulomb interaction potential

$$
v_{l}\left(k, k^{\prime}\right)=\frac{1}{2} \int_{0}^{\pi} d \theta \sin \theta P_{l}(\cos \theta)\left\langle\mathbf{k}|v| \mathbf{k}^{\prime}\right\rangle,
$$

that according to (2) can be written in the form

$$
v_{l}\left(k, k^{\prime}\right)=\frac{2 \pi q_{1} q_{2}}{k k^{\prime}} Q_{l}\left(\frac{k^{2}+k^{2}}{2 k k^{\prime}}\right),
$$

where the function $Q_{l}(x)$ is the Legendre polynomial of the second kind [22].

According to the definition (14), the partial wave component of the Coulomb transition matrix is equal to

$$
t_{l}\left(k, k^{\prime} ; E\right)=\frac{1}{2} \int_{0}^{\pi} d \theta \sin \theta P_{l}(\cos \theta)\left\langle\mathbf{k}|t(E)| \mathbf{k}^{\prime}\right\rangle .
$$

Taking the relationship of the angle between two 4-dimensional vectors in the fourdimensional Fock's space $\omega$ and the angle $\theta$ between the vectors $\mathbf{k}$ and $\mathbf{k}^{\prime}$, we find

$$
\cos \theta=\frac{\xi}{\eta}-\frac{1}{\eta} \sin ^{2} \frac{\omega}{2}=\frac{2 \xi-1+\cos \omega}{2 \eta},
$$

where

$$
\xi=\frac{\kappa^{2}\left(k^{2}+k^{\prime 2}\right)}{\left(k^{2}+\kappa^{2}\right)\left(k^{\prime 2}+\kappa^{2}\right)}, \quad \eta=\frac{2 \kappa^{2} k k^{\prime}}{\left(k^{2}+\kappa^{2}\right)\left(k^{\prime 2}+\kappa^{2}\right)} .
$$

Passing in (18) from the integration over the angle $\theta$ to the integration over the variable $\omega$, we write the expression (18) for the partial wave component of the Coulomb transition matrix in the form

$$
t_{l}\left(k, k^{\prime} ; E\right)=\frac{1}{4 \eta} \int_{\omega_{0}}^{\omega_{\pi}} d \omega \sin \omega P_{l}\left(\frac{2 \xi-1+\cos \omega}{2 \eta}\right)\left\langle\mathbf{k}|t(E)| \mathbf{k}^{\prime}\right\rangle,
$$

where the integration limits in (22) are determined by the expressions

$$
\omega_{0}=2 \arcsin \sqrt{\xi-\eta}, \quad \omega_{\pi}=2 \arcsin \sqrt{\xi+\eta},
$$

in this case

$$
\begin{array}{rlrl}
\sin \omega_{0}= & 2 \sqrt{\xi-\eta} \sqrt{1-\xi+\eta}, & \sin \omega_{\pi}=2 \sqrt{\xi+\eta} \sqrt{1-\xi-\eta} . \\
\cos \omega_{0}=1-2 \xi+2 \eta, & \cos \omega_{\pi}=1-2 \xi-2 \eta,
\end{array}
$$

Substituting the expression (3) for the three-dimensional transition matrix into (21), we obtain the formula for the partial wave Coulomb transition matrix $t_{l}\left(k, k^{\prime} ; E\right)$ at $E<0$ :

$$
\begin{gathered}
t_{l}\left(k, k^{\prime} ; E\right)=\frac{\pi q_{1} q_{2}}{k k^{\prime}} \int_{\omega_{0}}^{\omega_{\pi}} d \omega P_{l}\left(\frac{2 \xi-1+\cos \omega}{2 \eta}\right)\left\{\cot \frac{\omega}{2}\right. \\
-\pi \gamma \cos \gamma \omega-\gamma \sin 2 \gamma \omega \ln \left(\sin \frac{\omega}{2}\right)+2 \pi \gamma c(\gamma) \cot \gamma \pi \sin \gamma \omega \\
\left.+\gamma \cos \gamma \omega x_{\gamma}(\omega)+2 \gamma^{2} \sin \gamma \omega y_{\gamma}(\omega)\right\} .
\end{gathered}
$$




\section{Partial wave Coulomb transition matrices at the energy of the excited state}

It is easy to see that the expression (24) for the partial wave Coulomb t-matrix is significantly simplified, if the energy $E$ is equal to the values of the bound states energies (7), $E=E_{n}$, at which the parameter $\gamma$ takes the integer values. (Note that in the case of the attractive interaction $\left(q_{1} q_{2}<0\right)$ this statement is relevant only for the partial wave transition matrices with the orbital momenta $l \geq n$, which have no pole singularities at these values of the energy.)

At $\gamma= \pm 1$, that corresponds to the bound ground state energy $E_{1}$, the functions (6) have a simple form

$$
x_{1}(\omega)=\omega+\sin \omega, \quad y_{1}(\omega)=\cos ^{2} \frac{\omega}{2}+2 \sin ^{2} \frac{\omega}{2} \ln \sin \left(\frac{\omega}{2}\right),
$$

in this case

$$
x_{-1}(\omega)=-x_{1}(\omega) \quad y_{-1}(\omega)=-y_{1}(\omega),
$$

This ensures a possibility to obtain the analytical expression for the partial wave Coulomb transition matrices at the energy $E_{1}$ for both the attractive [20] and repulsive [21] Coulomb interactions.

In this paper we extend the above results for the partial wave transition matrices at the ground- state energy to a case when the variable of the energy $E$ takes the value of the energy of the first excited state $E_{2}$. Then the functions $x_{2}(\omega)$ and $y_{2}(\omega)$ in the expression (24) assume the form

$$
x_{2}(\omega)=\omega+2 \sin \omega+\frac{1}{2} \sin 2 \omega, \quad y_{2}(\omega)=-\cos ^{4} \frac{\omega}{2}-\sin ^{2} \omega \ln \sin \left(\frac{\omega}{2}\right),
$$

In the case of the repulsive Coulomb interaction $\left(q_{1} q_{2}>0\right.$, inserting (26) into the formula for the partial wave Coulomb transition matrix (24) at $E=E_{2}(\gamma=2)$ and using (10) - (12), we obtain

$$
\begin{aligned}
t_{l}^{r}\left(k, k^{\prime} ; E\right) & =\frac{\pi q_{1} q_{2}}{k k^{\prime}} \int_{\omega_{0}}^{\omega_{\pi}} d \omega P_{l}\left(\frac{2 \xi-1+\cos \omega}{2 \eta}\right)\left\{\cot \frac{\omega}{2}\right. \\
& -2 \pi \cos 2 \omega+2 \omega \cos 2 \omega-4 \sin \omega \\
+ & \left.\left(\rho_{2}-3\right) \sin 2 \omega-4 \sin 2 \omega \ln \left(\frac{\omega}{2}\right)\right\},
\end{aligned}
$$

where according to $(12)$

$$
\rho_{2}=3-4 \ln 2 .
$$

In the case of the attractive interaction $\left(q_{1} q_{2}<0\right.$, the formula for the partial wave Coulomb transition matrix $(24)$ at $E=E_{2}(\gamma=-2)$ takes the form

$$
\begin{aligned}
t_{l}^{a}\left(k, k^{\prime} ; E\right)= & \frac{\pi q_{1} q_{2}}{k k^{\prime}} \int_{\omega_{0}}^{\omega_{\pi}} d \omega P_{l}\left(\frac{2 \xi-1+\cos \omega}{2 \eta}\right)\left\{\cot \frac{\omega}{2}\right. \\
+ & 2 \pi \cos 2 \omega-2 \omega \cos 2 \omega-4 \sin \omega \\
& \left.-3 \sin 2 \omega-4 \sin 2 \omega \ln \left(\frac{\omega}{2}\right)\right\},
\end{aligned}
$$

Herewith, it must be remembered that the singularities in the energy of the threedimensional Coulomb transition matrix for the attractive interaction in (3) pass only to the partial wave transition matrices with the orbital momenta $l<n$. All other partial 
wave components of the transition matrix are nonsingular at $E=E_{n}$. This follows from the form of the fourth term in (24) which contains the coefficient

$$
I_{\gamma l} \equiv \int_{\omega_{0}}^{\omega_{\pi}} d \omega \sin \gamma \omega P_{l}\left(\frac{2 \xi-1+\cos \omega}{2 \eta}\right)
$$

before the singular term $\cot \gamma \pi$. In the case $\gamma=-2$, which is under study, for example, we have

$$
I_{-2 l}=-\frac{16}{3} \eta^{2} \delta_{l 1}+8 \eta(2 \xi-1) \delta_{l 0} .
$$

In this way, all the partial wave transition matrices with the orbital momenta $l \geq 2$ have no pole singularities.

5. Analytical expressions for the partial wave transition matrices at the energy $E=E_{2}$ in the case of the repulsive Coulomb interaction $\left(q_{1} q_{2}>0\right)$

Taking the integration over $\omega$ in the expression (27 we obtain simple analytical expressions for the partial wave Coulomb $t$-matrix at $E=E_{2}$ in the case of like charges $(\gamma=2)$. In particular, the expression for the partial $s$-wave Coulomb t-matrix in the case of the repulsive interaction has the form

$$
\begin{gathered}
t_{0}^{r}\left(k, k^{\prime} ; E_{2}\right)=\frac{\pi q_{1} q_{2}}{k k^{\prime}}\left\{16(3 \xi-2) \eta-8(2 \xi-1) \eta \rho_{2}+\left(8 \xi^{2}-8 \xi+8 \eta^{2}+1\right) \ln \frac{\xi+\eta}{\xi-\eta}\right. \\
\left.+8(2 \xi-1) \eta \ln \left(\xi^{2}-\eta^{2}\right)-\left(\pi-\omega_{\pi}\right) \sin 2 \omega_{\pi}+\left(\pi-\omega_{0}\right) \sin 2 \omega_{0}\right\} .
\end{gathered}
$$

Similarly we get the analytical expressions for the partial $p$ - and $d$ - wave Coulomb $t$-matrices:

$$
\begin{gathered}
t_{1}^{r}\left(k, k^{\prime} ; E_{2}\right)=\frac{\pi q_{1} q_{2}}{k k^{\prime}}\left\{\left(\frac{8}{3} \xi-16 \eta^{2}-2\right)+\frac{16}{3} \eta^{2} \rho_{2}\right. \\
+\frac{1}{\eta}\left(\frac{8}{3} \xi^{3}-4 \xi^{2}+\xi-8 \xi \eta^{2}+4 \eta^{2}\right) \ln \frac{\xi+\eta}{\xi-\eta}-\frac{16}{3} \eta^{2} \ln \left(\xi^{2}-\eta^{2}\right) \\
-\frac{1}{2 \eta}\left[\left(\pi-\omega_{\pi}\right) \sin \omega_{\pi}-\left(\pi-\omega_{0}\right) \sin \omega_{0}\right] \\
-\frac{1}{2 \eta}(2 \xi-1)\left[\left(\pi-\omega_{\pi}\right) \sin 2 \omega_{\pi}-\left(\pi-\omega_{0}\right) \sin 2 \omega_{0}\right] \\
\left.-\frac{1}{6 \eta}\left[\left(\pi-\omega_{\pi}\right) \sin 3 \omega_{\pi}-\left(\pi-\omega_{0}\right) \sin 3 \omega_{0}\right]\right\}
\end{gathered}
$$

and

$$
\begin{gathered}
t_{2}^{r}\left(k, k^{\prime} ; E_{2}\right)=\frac{\pi q_{1} q_{2}}{k k^{\prime}}\left\{\frac{1}{\eta}\left(2 \xi^{2}-\frac{5}{2} \xi-2 \eta^{2}+\frac{3}{4}\right)\right. \\
+\frac{1}{\eta^{2}}\left[\left(-2 \xi^{4}+4 \xi^{3}+\frac{3}{2} \xi^{2}+4 \xi^{2} \eta^{2}+12 \xi \eta^{2}-\frac{1}{2} \eta^{2}\right)-2 \eta^{4}\right) \ln \frac{\xi+\eta}{\xi-\eta} \\
-\frac{3 \pi}{16}\left(\omega_{\pi}-\omega_{0}\right)+\frac{3}{32}\left(\omega_{\pi}^{2}-\omega_{0}^{2}\right)-\frac{3}{4}(2 \xi-1)\left[\left(\pi-\omega_{\pi}\right) \sin \omega_{\pi}-\left(\pi-\omega_{0}\right) \sin \omega_{0}\right] \\
-\left(\frac{3}{2} \xi^{2}-\frac{3}{2} \xi-\frac{1}{2} \eta^{2}+\frac{9}{16}\right)\left[\left(\pi-\omega_{\pi}\right) \sin 2 \omega_{\pi}-\left(\pi-\omega_{0}\right) \sin 2 \omega_{0}\right] \\
-\frac{1}{4}(2 \xi-1)\left[\left(\pi-\omega_{\pi}\right) \sin 3 \omega_{\pi}-\left(\pi-\omega_{0}\right) \sin 3 \omega_{0}\right] \\
\left.\left.-\frac{3}{64}\left[\left(\pi-\omega_{\pi}\right) \sin 4 \omega_{\pi}-\left(\pi-\omega_{0}\right) \sin 4 \omega_{0}\right]\right]\right\}
\end{gathered}
$$


Note that the formulae for $\xi$ and $\eta(20)$, as well as the formulae for $\omega_{0}$ and $\omega_{\pi}$ contain $\kappa=\kappa_{2}$, corresponding to the energy $E_{2}(7)$.

6. Analytical expressions for the partial wave transition matrix at the energy $E=E_{2}$ in the case of the attractive Coulomb interaction $\left(q_{1} q_{2}<0\right)$

As is started at the end of Section 4, according to (31), the partial wave components of the transition matrices for the oppositely charged particles $t_{l}^{a}\left(k, k^{\prime} ; E\right)$ with $l=0$ and $l=1$ are singular at the point $E=E_{2}$. The lowest nonsingular at this point partial-wave component is the $d$-wave component with $l=2$. Taking the corresponding integration over $\omega$ in the expression (29) $l=2$ we obtain the following formula for $t_{2}^{a}\left(k, k^{\prime} ; E_{2}\right)$ :

$$
\begin{gathered}
t_{2}^{a}\left(k, k^{\prime} ; E_{2}\right)=\frac{\pi q_{1} q_{2}}{k k^{\prime}}\left\{\frac{1}{\eta}\left(-8 \xi^{3}+14 \xi^{2}-\frac{7}{2} \xi+\frac{40}{3} \xi \eta^{2}-\frac{26}{3} \eta^{2}-\frac{3}{4}\right)\right. \\
+\frac{1}{\eta^{2}}\left[\left(-2 \xi^{4}+4 \xi^{3}+\frac{3}{2} \xi^{2}+4 \xi^{2} \eta^{2}+12 \xi \eta^{2}-\frac{1}{2} \eta^{2}\right)-2 \eta^{4}\right) \ln \frac{\xi+\eta}{\xi-\eta} \\
+\frac{3 \pi}{16}\left(\omega_{\pi}-\omega_{0}\right)-\frac{3}{32}\left(\omega_{\pi}^{2}-\omega_{0}^{2}\right)+\frac{3}{4}(2 \xi-1)\left[\left(\pi-\omega_{\pi}\right) \sin \omega_{\pi}-\left(\pi-\omega_{0}\right) \sin \omega_{0}\right] \\
+\left(\frac{3}{2} \xi^{2}-\frac{3}{2} \xi-\frac{1}{2} \eta^{2}+\frac{9}{16}\right)\left[\left(\pi-\omega_{\pi}\right) \sin 2 \omega_{\pi}-\left(\pi-\omega_{0}\right) \sin 2 \omega_{0}\right] \\
+\frac{1}{4}(2 \xi-1)\left[\left(\pi-\omega_{\pi}\right) \sin 3 \omega_{\pi}-\left(\pi-\omega_{0}\right) \sin 3 \omega_{0}\right] \\
\left.\left.+\frac{3}{64}\left[\left(\pi-\omega_{\pi}\right) \sin 4 \omega_{\pi}-\left(\pi-\omega_{0}\right) \sin 4 \omega_{0}\right]\right]\right\}
\end{gathered}
$$

It is interesting to note, taking into account the difference in sign for $q_{1} q_{2}$ in the coefficients before the braces in the expressions (34) and (35), that the formulae for the corresponding partial $d$-wave transition matrices in the cases of the repulsive and attractive Coulomb interactions differ only by their first terms and the signs in front the terms with the logarithm. The remaining terms are the same.

\section{Discussion and conclusions}

The investigation of the properties of the off-energy-shell two-body partial wave Coulomb transition matrices is necessary in connection with the formulation and solution of the integral equations for composite atomic and nuclear systems.

The possibility of the analytical derivation of the two-body partial wave Coulomb transition matrices with the use of the specific symmetry of the Coulomb system in Fock's four-dimensional Euclidean space has been first studied in our preceding paper [20]. Therein the simple analytical expressions for the partial $p$-, $d$ - and $f$-wave Coulomb transition matrices at the energy of the qround bound state $E=E_{1}$ (that corresponds to the Coulomb parameter $\gamma=-1$ ) have been derived in the case of the oppositely charged particles. In the case of the repulsive Coulomb potential analytical expressions for the partial $s$-, $p$ - and $d$-wave transition matrices at the energy $E=E_{1}(\gamma=1)$ have been obtained in Ref. [21].

In this paper the Fock's method has been applied to derive the partial wave Coulomb transition matrices at the first excited state energy $E=E_{2}$. The cases of the two-body system both with repulsive and attractive Coulomb interactions have been investigated (the transition matrices $t_{l}^{r}\left(k, k^{\prime} ; E_{2}\right)$ and $\left.t_{l}^{a}\left(k, k^{\prime} ; E_{2}\right)\right)$. It is interesting that the simplification of the analytical form of the partial wave transition matrix at the energies 
of the bound states takes place both for the particles with opposite charges and for the particles with like charges, bound states of which are not formed at all.

The knowledge of partial wave Coulomb transition matrix is necessary in particular to determine the electric $2^{\lambda}$-pole polarizabilities of the two-particle Coulomb system $\alpha_{\lambda}$ $(\lambda=1,2 \cdots)$ in the state with the energy $E_{n}[23]$.

For example, in the case of the hydrogen-like atom with an infinite-mass nucleus we write the expression for the electric $2^{\lambda}$-pole polarizabilities $\alpha_{E \lambda}(\lambda=1,2,3, \ldots)$ in terms of the wave function, its corresponding derivatives and the partial wave transition matrix $t_{\lambda}$ at the energy of the first excited bound state $\left(n=2, l=0, E_{2}=-b_{2}\right)$ in the form

$$
\begin{gathered}
\alpha_{E \lambda}=\frac{2}{(2 \lambda+1) \pi^{2}} \frac{m_{1} q_{1}^{2}}{\hbar^{2}}\left\{\int_{0}^{\infty} d k k^{2} \frac{\left|\varphi_{\lambda}(k)\right|^{2}}{k^{2}+\kappa_{2}^{2}}\right. \\
\left.-\frac{m_{1}}{\pi^{2} \hbar^{2}} \int_{0}^{\infty} d k k^{2} \int_{0}^{\infty} d k^{\prime} k^{\prime 2} \frac{\varphi_{\lambda}^{*}(k) t_{\lambda}\left(k, k^{\prime} ;-b_{2}\right) \varphi_{\lambda}\left(k^{\prime}\right)}{\left(k^{2}+\kappa_{2}^{2}\right)\left(k^{\prime 2}+\kappa_{2}^{2}\right)}\right\},
\end{gathered}
$$

where $m_{1}$ and $q_{1}$ are the mass and the charge of the electron,

$$
\varphi_{\lambda}(k)=(-1)^{\lambda} k^{\lambda}\left[\left(\frac{1}{k} \frac{d}{d k}\right)^{\lambda} \psi(k)\right],
$$

$\psi(k)$ is the wave function of the $S$-wave first excited bound state of the atom in the momentum space, $\kappa_{2}=m_{1}\left|q_{1} q_{2}\right| / 2 \hbar^{2}$.

The circumstance, that the Coulomb t-matrix at the energy of the bound state in (36) can be found by means of direct solving of the corresponding Lippmann-Schwinger integral equation in a simple analytical form, permits to obtain the analytical expression also for the electric multipole polarizabilities [24].

At present it is essentially interesting to study the possibility of derivation of expressions for the partial wave transition matrix on the basis of the one-parameter integral representation of the three-dimensional Coulomb transition matrix, proposed by Schwinger [13]. Also, of special importance is the analytical solution of the integral LippmannSchwinger equation for the partial wave Coulomb transition matrices with the values of the energy $E$ differing from the energies of both the ground and excited bound states, for example, in the case of the half-integer values of the Sommerfeld parameter, $\gamma=+1 / 2$ and $\gamma=-1 / 2$, when all the partial wave components are singularity-free in the energy.

\section{Acknowledgment}

The present work was partially supported by the National Academy of Sciences of Ukraine (project No. 0117U00237) and by the Program of Fundamental Research of the Department of Physics and Astronomy of NASU (project No. 0117U00240).

\section{References}

[1] J. C. Y. Chen and A. C. Chen. Advances in Atomic and Molecular Physics. Vol 8. Ed. D B Bates and I Estermann. Academic Press, New York - London. 1972. p. 71.

[2] L D Faddeev. Sov. Phys. JETP 12, 1014 (1961).

[3] L D Faddeev. Mathematical Aspects of the Three-Body Problem in the Quantum Scattering Theory. Israel Program of Sci. Transl., Jerusalem. 1965.

[4] O. A. Yakubovsky. Sov. J. Nucl. Phys. 5, 937 (1967).

[5] A. M. Vesselova. Teor. Mat. Fiz. 3, 326 (1970). 
[6] A. M. Vesselova. Teor. Mat. Fiz. 13, 368 (1972).

[7] V. G. Gorshkov. Zh. Eksp. Teor. Fiz. 40, 1481 (1961).

[8] S. Okubo and D. Feldman. Phys. Rev. 117, 292 (1960).

[9] E. H. Wichmann and C. H. Woo. J. Math. Phys. 2, 178 (1961).

[10] V. F. Bratsev and E. D. Trifonov. Vest. Leningrad. Gos. Univ. 16, 36 (1962).

[11] L. Hostler. J. Math. Phys. 5, 591 (1964).

[12] L. Hostler. J. Math. Phys. 5, 1235 (1964).

[13] J. Schwinger. J. Math. Phys. 5, 1606 (1964).

[14] A. M. Perelomov and V. S. Popov. Sov. Phys. JETP 23, 118 (1966).

[15] S. A. Shadchin and V. F. Kharchenko. J. Phys. B: At. Mol.Phys. 161319 (1983).

[16] S. A. Storozhenko and S. A. Shadchin. Teor. Mat. Fiz. 76339 (1988).

[17] L. P. Kok and H. van Haeringen. Phys. Rev. C, 21, 512 (1980).

[18] H. van Haeringen. J. Math. Phys. 25, 3001 (1984).

[19] V. A. Fock. Z. Phys. 98, 145 (1935).

[20] V. F. Kharchenko. Ann. Phys. NY. 374, 16 (2016).

[21] V. F. Kharchenko. Ukr. J. Phys. 62, 263 (2017).

[22] I. S. Gradstein and I. M. Ryzhik. Tables of Integrals, Sums,Series and Products. Nauka, Moscow, 1971.

[23] V. F. Kharchenko. J. Mod. Phys. 4, 99 (2013).

[24] V. F. Kharchenko. Ann. Phys. NY. 355, 153 (2015). 